\title{
SYNTESIS AND ANTIBACTERIAL ACTIVITY OF DIBENZYLIDENE-CYCLOHEXANONE
}

\author{
Ritmaleni $^{1,2,}$, A.N.A. Hastutitama ${ }^{2}$, I. Persitamaia ${ }^{2}$, T. Restiwardani ${ }^{2}$, \\ A. Eksakta ${ }^{2}$, R.F. Munandar ${ }^{2}$, M.S. Abdullah ${ }^{2}$, A.E. Purwanto ${ }^{2}$, P. Astuti ${ }^{3}$ \\ and Sardjiman ${ }^{1}$ \\ ${ }^{1}$ Department of Pharmaceutical Chemistry, Faculty of Pharmacy/Gadjah Mada University, \\ Yogyakarta-55281, Indonesia \\ ${ }^{2}$ Curcumin Research Center, Faculty of Pharmacy/Gadjah Mada University, Yogyakarta-55281, \\ Indonesia \\ ${ }^{3}$ Department of Biological Pharmacy, Faculty of Pharmacy/Gadjah Mada University, \\ Yogyakarta-55281, Indonesia \\ Corresponding Author: ritmaleni@ugm.ac.id
}

\begin{abstract}
Dibenzylidene-cyclohexanone was synthesized by reacting aromatic aldehydes with cyclohexanone in the acidic condition through the carbonyl condensation reaction. The antibacterial activity was tested by using microdilution method against K. pneumonia, E. coli, S. aureus, B. subtilis and E. faecalis. The isolated products were obtained as pure curcumin analogs with moderate to high yield. The antibacterial assay showed that 2,6-bis-(3'hydroxybenzylidene)-cyclohexanone gave MIC and MBC at $50 \mu \mathrm{g} / \mathrm{mL}$ in E. coli, S. aureus, and E. faecalis. Moreover, it showed that the percentage inhibition of B. subtilis was around $54 \%$ at a concentration of $25 \mu \mathrm{g} / \mathrm{mL}$. Among all the tested compounds, A146 showed better activity against all those bacteria while none showed activity against $K$. pneumonia. A111 was obtained as the compound with the highest yield and A146 was the most potent compound. While A102 was the most potent compound according to the docking study.

Keywords: Antibacterial Agent, Dibenzylidene-cyclohexanone, Docking Study, Synthesis

RASĀYAN J. Chem., Vol. 14, No.3, 2021
\end{abstract}

\section{INTRODUCTION}

The rush in finding new potent antimicrobials is a global issue at this time and the search for the new novel antimicrobial is urgently needed. ${ }^{1}$ Some studies showed that curcumin is effective against Staphylococcus aureus (S. aureus). ${ }^{2}$ Curcumin is also known as a golden compound and this compound has been studied for the treatment of diseases such as nervous system disorders ${ }^{3}$, antidepressant effect ${ }^{4}$ and epileptic rats ${ }^{5}$. Due to its lack of properties ${ }^{6}$, many curcumin analogs were synthesized ${ }^{7-9}$ and some have been developed as antibacterial agent ${ }^{10}$. In addition, an analog of curcumin has been also reported to have many biological activities such as anticancer, antimicrobial, antidiabetic, antioxidant, antiinflammatory, antituberculosis, and antileishmanial agents. ${ }^{11-17}$ Not only analog of curcumin that has been successfully synthesized, but derivative of curcumin also synthesized such as curcumin diclofenac which has been studied for its bioavailability. ${ }^{18-19}$ A series of curcumin derivatives containing heterocyclic moiety have been successfully synthesized and evaluated their antibacterial activities. ${ }^{20-21}$ From the previous study QSAR of antibacterial activity, against $K$. pneumoniae, B. subtilis, and $S$. aureus has also been reported. ${ }^{22}$ In continuation, some analogs from this series were also tested as antibacterial. ${ }^{23}$ Some heterocyclic and monocarbonyl of curcumin analog have been studied for their in silico and in vitro potency as antibacterial agents and other potential biological activities. ${ }^{24-28}$ This study is aimed to synthesize and study the antibacterial potency of curcumin analog, dibenzylidene-cyclohexanone.

\section{EXPERIMENTAL}

\section{Material and Method}

The main chemicals were aromatic aldehyde and cyclohexanone (Table-1). Other chemicals were organic solvents, $\mathrm{HCl}$, and $\mathrm{KMnO}_{4}$. The condensation reaction was performed in the synthesis process. The 
RASĀYAN J. Chem.

Vol. 14 | No. 3 |2090-2096| July - September | 2021

isolation and purification were carried out by the recrystallization technique. The structure of the pure compounds was determined by using the spectroscopic methods (IR, HNMR, CNMR and MS).

\section{General Procedure}

To a round bottom flask, aldehyde $(2 \mathrm{~mol}$. eq) and cyclohexanone $(1 \mathrm{~mol}$. eq) were added subsequently continued by THF. The reaction was catalyzed by $\mathrm{HCl}$. The reaction mixture was mixed at room temperature for 2 hours and then heated up at $20-50{ }^{\circ} \mathrm{C}$. The reaction was monitored by TLC with ethyl acetate: hexane $(1: 2 \mathrm{v} / \mathrm{v})$ as eluent and TLC was then stained with $\mathrm{KMnO}_{4}$. The product was isolated by washing the reaction mixture with ethanol: water $(1: 1 \mathrm{v} / \mathrm{v})$, followed by water. Recrystallization was carried out by using methanol/water.

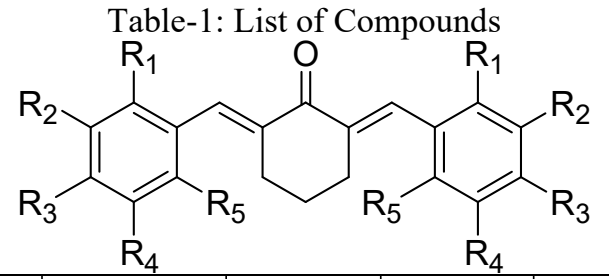

\begin{tabular}{c|c|c|c|c|c|c}
\hline No & Compound & $\mathrm{R}_{1}$ & $\mathrm{R}_{2}$ & $\mathrm{R}_{3}$ & $\mathrm{R}_{4}$ & $\mathrm{R}_{5}$ \\
\hline 1 & $\mathrm{~A} 102$ & $-\mathrm{OH}$ & $-\mathrm{OEt}$ & $-\mathrm{H}$ & $-\mathrm{H}$ & $-\mathrm{H}$ \\
\hline 2 & $\mathrm{~A} 103$ & $-\mathrm{H}$ & $-\mathrm{OEt}$ & $-\mathrm{OH}$ & $-\mathrm{H}$ & $-\mathrm{H}$ \\
\hline 3 & $\mathrm{~A} 104$ & $-\mathrm{H}$ & $-\mathrm{NO}_{2}$ & $-\mathrm{H}$ & $-\mathrm{H}$ & $-\mathrm{H}$ \\
\hline 4 & $\mathrm{~A} 108$ & $-\mathrm{H}$ & $-\mathrm{COH}$ & $-\mathrm{H}$ & $-\mathrm{H}$ & $-\mathrm{H}$ \\
\hline 5 & $\mathrm{~A} 111$ & $-\mathrm{OMe}$ & $-\mathrm{H}$ & $-\mathrm{H}$ & $-\mathrm{OMe}$ & $-\mathrm{H}$ \\
\hline 6 & $\mathrm{~A} 129$ & $-\mathrm{OMe}$ & $-\mathrm{H}$ & $-\mathrm{H}$ & $-\mathrm{H}$ & $-\mathrm{H}$ \\
\hline 7 & $\mathrm{~A} 146$ & $-\mathrm{H}$ & $-\mathrm{OH}$ & $-\mathrm{H}$ & $-\mathrm{H}$ & $-\mathrm{H}$ \\
\hline
\end{tabular}

Synthesis of 2,6-bis-(3'-ethoxy-2'-hydroxybenzylidene)-cyclohexanone, A102<smiles>CCOc1cccc(C=C2CCCC(=Cc3cccc(OCC)c3O)C2=O)c1O</smiles>

Brownish-yellow crystals; yield $40 \%$; m.p. $133.7-134.5{ }^{\circ} \mathrm{C}$ (Toluene : Hexane); IR $\left(\mathrm{cm}^{-1}, \mathrm{KBr}\right): 1580$, 2839-3474, 728, 1214, 1467, 1645, 3037, 2977, 1113, 944; ${ }^{1} \mathrm{H}-\mathrm{NMR}$ (500 MHz, ppm, Acetone): $\delta 2.90$ $\left(4 \mathrm{H}, \mathrm{m}, \mathrm{H}_{3,5}\right), 1.80\left(2 \mathrm{H}, \mathrm{m}, \mathrm{H}_{4}\right), 1.46\left(6 \mathrm{H}, \mathrm{m}, \mathrm{H}_{22,24}\right), 4.13\left(4 \mathrm{H}, \mathrm{m}, \mathrm{H}_{21,23}\right), 7.08\left(2 \mathrm{H}, \mathrm{dd}, J_{1}=7.5 \mathrm{~Hz}, J_{2}=1.5\right.$ $\left.\mathrm{Hz}, \mathrm{H}_{13,20}\right), 7.03\left(2 \mathrm{H}, \mathrm{dd}, J_{I}=7.5 \mathrm{~Hz}, J_{2}=7.5 \mathrm{~Hz}, \mathrm{H}_{12,19}\right), 6.93\left(\mathrm{dd}, J_{l}=7.5 \mathrm{~Hz}, J_{2}=1.5 \mathrm{~Hz}, \mathrm{H}_{11,18}\right), 5.85(2 \mathrm{H}$, $\left.\mathrm{s}, \mathrm{H}_{7,14}\right), 7.70(2 \mathrm{H}, \mathrm{s}, 2 \mathrm{x} \mathrm{OH}) ;{ }^{13} \mathrm{C}-\mathrm{NMR}\left(125 \mathrm{MHz}, \mathrm{ppm}\right.$, Acetone): $\delta 23$ (s), $28(\mathrm{~s}), 53\left(\mathrm{CH}_{3}\right), 64(\mathrm{~s}), 114$ $(\mathrm{t}), 124(\mathrm{t}), 128(\mathrm{t}), 134(\mathrm{t}), 137(\mathrm{t}), 145(\mathrm{q}), 146(\mathrm{q}), 190(\mathrm{C}=\mathrm{O}) ; \mathrm{MS}(\mathrm{EI}-\mathrm{MS}, \mathrm{m} / \mathrm{z}, \%): 394\left(\left[\mathrm{M}^{+}\right], 5\right)$, 377(100), 347(23), 319(15), 228(65), 197(18), 123(23), 77(15), 55(24). Obtained as $\mathrm{C}_{24} \mathrm{H}_{26} \mathrm{O}_{5}$

\section{Synthesis of 2,6-bis-(3'-ethoxy-4'-hydroxybenzylidene)-cyclohexanone, A103}

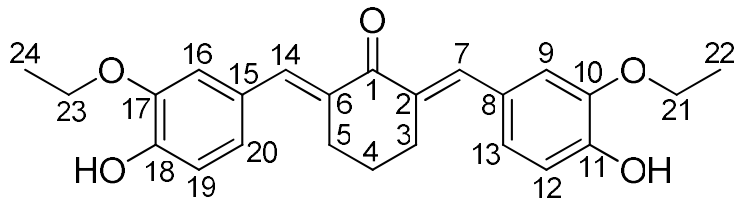

Yellow crystals; yield 73 \%; m.p. $156.8-157.6{ }^{\circ} \mathrm{C}$ (DCM : Hexane); IR (cm $\left.{ }^{-1}, \mathrm{KBr}\right) 3530,3345,3139$, 2873, 1654, 1591, 1506, 1475, 1252, 1036, 1214; ${ }^{1} \mathrm{H}-\mathrm{NMR}\left(500 \mathrm{MHz}, \mathrm{ppm}, \mathrm{CDCl}_{3}\right): \delta 1.45(2 \mathrm{H}, \mathrm{t}, J=6$ $\left.\mathrm{Hz}, \mathrm{H}_{22,24}\right), 1.79\left(2 \mathrm{H}, \mathrm{q}, J=5 \mathrm{~Hz}, \mathrm{H}_{4}\right), 2.90\left(2 \mathrm{H}, \mathrm{t}, J=5 \mathrm{~Hz}, \mathrm{H}_{3,5}\right), 4.11,\left(2 \mathrm{H}, \mathrm{q}, J=6 \mathrm{~Hz}, \mathrm{H}_{21,23}\right), 5.85(2 \mathrm{H}$ , s, $\left.\mathrm{H}_{7}, 14\right), 6.94\left(2 \mathrm{H}, \mathrm{d}, J=8 \mathrm{~Hz}, \mathrm{H}_{12}, 19\right), 6.96\left(2 \mathrm{H}, \mathrm{d}, J=2 \mathrm{~Hz}, \mathrm{H}_{13,18}\right), 7.04\left(2 \mathrm{H}, \mathrm{dd}, J_{l}=2 \mathrm{~Hz}, J_{2}=8 \mathrm{~Hz}\right.$, $\left.\mathrm{H}_{9,16}\right), 7.70(2 \mathrm{H}, \mathrm{s}, 2 \mathrm{x} \mathrm{OH}) ;{ }^{13} \mathrm{C}-\mathrm{NMR}\left(500 \mathrm{MHz}, \mathrm{ppm}, \mathrm{CDCl}_{3}\right): \delta 15\left(\mathrm{CH}_{3}\right), 23(\mathrm{~s}), 28(\mathrm{~s}), 64(\mathrm{~s}), 114(\mathrm{t})$, $124(\mathrm{t}), 128(\mathrm{t}), 134$ (t), 137 (t), 145 (q), 146 (q), 190 (C=O); MS (EI-MS, m/z, \%): $394\left(\mathrm{M}^{+*}, 100\right), 366(40)$, 337(30), 291(25), 115(45), 102(38), 91(35), 77(40), 65(30), 55(60), 43(25) Obtained as $\mathrm{C}_{24} \mathrm{H}_{26} \mathrm{O}_{5}$. 
RASĀYAN J. Chem.

Vol. 14 | No. 3 |2090-2096| July - September | 2021

\section{Synthesis of 2,6-bis-(3'-nitrobenzylidene)-cyclohexanone, A104}

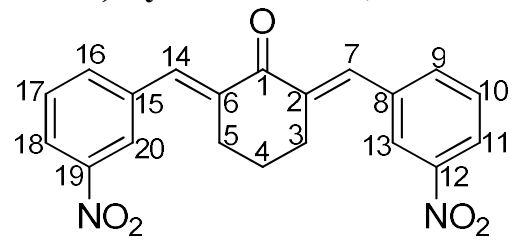

Yellow crystals; yield 54 \%; m.p. $194.3-194.9^{\circ} \mathrm{C}\left(\mathrm{CHCl}_{3}\right.$ : Hexane); IR (cm $\left.{ }^{-1}, \mathrm{KBr}\right) 3152,3013,1663$, 1604, 1573, 1518, 1350, 707; ${ }^{1} \mathrm{H}-\mathrm{NMR}\left(500 \mathrm{MHz}, \mathrm{ppm}, \mathrm{CDCl}_{3}\right): \delta 1.85\left(2 \mathrm{H}, \mathrm{q}, J=6.5 \mathrm{~Hz}, \mathrm{H}_{4}\right), 2.95(4 \mathrm{H}$, $\left.\mathrm{t}, J=6.5 \mathrm{~Hz}, \mathrm{H}_{3,5}\right), 7.59\left(2 \mathrm{H}, \mathrm{t}, J=8 \mathrm{~Hz}, \mathrm{H}_{10,17}\right), 7.73\left(2 \mathrm{H}, \mathrm{d}, J=8 \mathrm{~Hz}, \mathrm{H}_{9,16}\right), 7.79\left(2 \mathrm{H}, \mathrm{s}, \mathrm{H}_{7,14}\right), 8.19(2 \mathrm{H}$, d, $\left.J=8 \mathrm{~Hz}, \mathrm{H}_{11,18}\right), 8.30\left(2 \mathrm{H}, \mathrm{s}, \mathrm{H}_{13,20}\right) ;{ }^{13} \mathrm{C}-\mathrm{NMR}\left(125 \mathrm{MHz}, \mathrm{ppm}, \mathrm{CDCl}_{3}\right): \delta 22(\mathrm{~s}) ; 28(\mathrm{~s}) ; 123(\mathrm{t}) ; 129(\mathrm{t}) ;$ $134(\mathrm{t}) ; 136(\mathrm{t}) ; 137(\mathrm{t}) ; 138(\mathrm{t}) ; 148(\mathrm{q}) ; 189$ (C=O); MS (EI-MS, m/z) $364\left(\mathrm{M}^{+*}, 19\right) ; 347$ (100); 336(13), 317(38), 289(25), 215(30); 161(19), 115(70), 89(38), 63(40), 51(34), 39(31). Obtained as $\mathrm{C}_{20} \mathrm{H}_{16} \mathrm{~N}_{2} \mathrm{O}_{5}$.

\section{Synthesis of 2,6-bis-(3'-formylbenzylidene)-cyclohexanone, A108}<smiles>O=Cc1cccc(C=C2CCCC(=Cc3ccccc3)C2=O)c1</smiles>

Yellow crystals; yield 27 \%; m.p. $220.6-221.4^{\circ} \mathrm{C}(\mathrm{DCM})$; IR $\left(\mathrm{cm}^{-1}, \mathrm{KBr}\right) 3043,2943,2872,2726,2837$, $1695,1658,1597,1560,1505,1413,826,790 ;{ }^{1} \mathrm{H}-\mathrm{NMR}\left(500 \mathrm{MHz}, \mathrm{ppm}, \mathrm{CDCl}_{3}\right): \delta 1.84(2 \mathrm{H}, \mathrm{q}, J=5.5$ $\left.\mathrm{Hz}, \mathrm{H}_{4}\right), 2.95\left(2 \mathrm{H}, \mathrm{t}, J=5.5 \mathrm{~Hz}, \mathrm{H}_{3}\right), 2.99\left(2 \mathrm{H}, \mathrm{t}, J=5.5 \mathrm{~Hz}, \mathrm{H}_{5}\right), 7.54\left(2 \mathrm{H}, \mathrm{s}, \mathrm{H}_{9,16}\right), 7.61(2 \mathrm{H}, \mathrm{d}, J=8 \mathrm{~Hz}$, $\left.\mathrm{H}_{13,20}\right), 7.81\left(2 \mathrm{H}, \mathrm{m}, \mathrm{H}_{12,19}\right), 7.93\left(2 \mathrm{H}, \mathrm{d}, J=8 \mathrm{~Hz}, \mathrm{H}_{11,18}\right), 10.04\left(2 \mathrm{H}, \mathrm{s}, \mathrm{H}_{21,22}\right) ;{ }^{13} \mathrm{C}-\mathrm{NMR}(125 \mathrm{MHz}, \mathrm{ppm}$, $\left.\mathrm{CDCl}_{3}\right): \delta 22(\mathrm{~s}) ; 28(\mathrm{~s}) ; 129(\mathrm{t}) ; 130(\mathrm{t}) ; 130(\mathrm{t}) ; 135(\mathrm{t}) ; 135(\mathrm{t}) ; 136(\mathrm{t}) ; 136(\mathrm{t}) ; 136(\mathrm{t}) ; 138(\mathrm{t}) ; 141(\mathrm{q}) ;$ $189(\mathrm{C}=\mathrm{O}) ; 191(\mathrm{C}=\mathrm{O}) ; \mathrm{MS}(\mathrm{EI}-\mathrm{MS}, \mathrm{m} / \mathrm{z}) 330\left(\mathrm{M}^{+\bullet}, 100\right) ; 301(70) ; 217(30) ; 202(8) ; 128$ (45);115 (74); 77 (30); 57 (28); 43 (18). Obtained as $\mathrm{C}_{22} \mathrm{H}_{18} \mathrm{O}_{3}$.

\section{Synthesis of 2,6-bis-(2',5'-dimethoxybenzylidene)-cyclohexanone, A111}

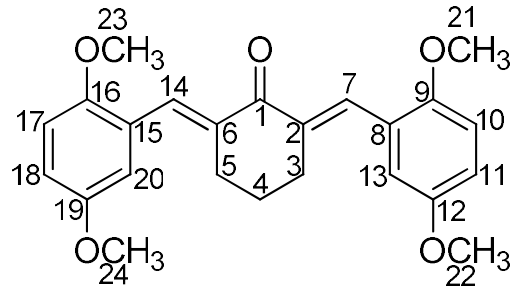

Yellow crystals; yield $81 \%$; m.p. 143.0-143.1 ${ }^{\circ} \mathrm{C}(\mathrm{EtOH})$; IR (cm $\left.{ }^{-1}, \mathrm{KBr}\right): 2947,2831,1662,1600,1583$, $1488,1461,1369,1442,1249,1033,933,864,810 ;{ }^{1} \mathrm{H}-\mathrm{NMR}\left(500 . \mathrm{MHz}, \mathrm{ppm}, \mathrm{CDCl}_{3}\right): \delta 1.73\left(2 \mathrm{H}, m, \mathrm{H}_{10}\right)$, $2.82\left(4 \mathrm{H}, m, \mathrm{H}_{9,10}\right), 3.76\left(6 \mathrm{H}, s, 2 \mathrm{x}-\mathrm{OCH}_{3}\right), 3.77\left(6 \mathrm{H}, s, 2 \mathrm{x}-\mathrm{OCH}_{3}\right), 6.81\left(2 \mathrm{H}, \mathrm{m}, \mathrm{H}_{10,17}\right), 6.83\left(2 \mathrm{H}, s, \mathrm{H}_{13,20}\right)$, $6.85\left(2 \mathrm{H}, m, \mathrm{H}_{11,18}\right), 7.91\left(2 \mathrm{H}, s, \mathrm{H}_{7,14}\right),{ }^{13} \mathrm{C}-\mathrm{NMR}\left(500 \mathrm{MHz}, \mathrm{ppm}, \mathrm{CDCl}_{3}\right): \delta 23(\mathrm{~s}), 28(\mathrm{~s}), 56\left(-\mathrm{OCH}_{3}\right), 111$ (t), $114(\mathrm{t}), 116(\mathrm{t}), 126(\mathrm{t}), 132(\mathrm{t}), 137$ (t), 152 (q), 190 (C=O); MS (EI-MS, m/z, \%): 394(M $\left.{ }^{+\bullet}, 8\right), 364(30)$, 363(100). Obtained as $\mathrm{C}_{24} \mathrm{H}_{26} \mathrm{O}_{5}$

\section{Synthesis of 2,6-bis-(2'-methoxybenzylidene)-cyclohexanone, A129}

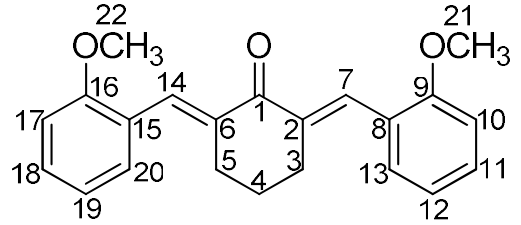

Yellow crystals; yield 74 \%; m.p. 143.7 - $143.8^{\circ} \mathrm{C}(\mathrm{EtOH})$; IR (cm $\left.{ }^{-1}, \mathrm{KBr}\right): 3060,2930,1664,1612,1597$, 1484, 1241, 1022, 943, 749; ${ }^{1} \mathrm{H}-\mathrm{HNMR}\left(500 \mathrm{MHz}, \mathrm{ppm}, \mathrm{CDCl}_{3}\right): \delta 1.72\left(2 \mathrm{H}, m ; \mathrm{H}_{4}\right), 2.82\left(4 \mathrm{H}, m ; \mathrm{H}_{3,5}\right)$, $3.84\left(6 \mathrm{H}, s, 2 \times \mathrm{OCH}_{3}\right), 6.90\left(2 \mathrm{H}, d, J=8.5, \mathrm{H}_{10,17}\right), 6.94\left(2 \mathrm{H}, d d, J_{1}=7 \mathrm{~Hz} ; J_{2}=7.5 \mathrm{~Hz} ; \mathrm{H}_{12,19}\right), 7.30(4 \mathrm{H}$, $\left.d d, J_{l}=J_{2}=7.5 \mathrm{~Hz}, \mathrm{H}_{11,18}, \mathrm{H}_{13,20}\right), 7.95\left(2 \mathrm{H}, s, \mathrm{H}_{7,14}\right) ;{ }^{13} \mathrm{C}-\mathrm{NMR}\left(500 \mathrm{MHz}, \mathrm{ppm}, \mathrm{CDCl}_{3}\right): \delta 23(\mathrm{~s}), 28(\mathrm{~s})$, 
$55(\mathrm{~s}), 110(\mathrm{t}), 120(\mathrm{t}), 125(\mathrm{t}), 130(\mathrm{t}), 130(\mathrm{t}), 132(\mathrm{t}), 136(\mathrm{t}), 158(\mathrm{q}), 190(\mathrm{q}) ;$ MS (EI-MS, m/z, \%) : 334( $\left.\mathrm{M}^{+\bullet}, 3\right), 303(100), 271(10), 197(22), 131(25), 115(35), 103(10), 91(30), 77(24), 51(10), 39(11)$; Obtained as $\mathrm{C}_{22} \mathrm{H}_{22} \mathrm{O}_{3}$

Synthesis of 2,6-bis-(3'-hydroxybenzylidene)-cyclohexanone, A146

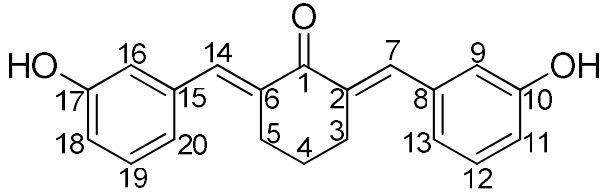

Yellow crystals; yield $58 \%$; m.p. $214.1-215.2{ }^{\circ} \mathrm{C}\left(\mathrm{CH}_{3} \mathrm{OH}: \mathrm{H}_{2} \mathrm{O}\right)$; IR ( $\left.v_{\max }, \mathrm{cm}^{-1}, \mathrm{KBr}\right): 3331,3182,2939$, $1650,1599,1445,1349,1333,1219,688,794,878 ; 1 \mathrm{H}-\mathrm{NMR}\left(500 \mathrm{MHz}, \mathrm{ppm}, \mathrm{DMSO}-\mathrm{d}_{6}\right): \delta 1.71(2 \mathrm{H}, \mathrm{m}$, $\left.\mathrm{H}_{4}\right), 2.87\left(4 \mathrm{H}, \mathrm{m}, \mathrm{H}_{3,5}\right), 6.79\left(2 \mathrm{H}, \mathrm{dd}, J_{l}=8 \mathrm{~Hz} ; J_{2}=2 \mathrm{~Hz}, \mathrm{H}_{13,20}\right), 6.92\left(2 \mathrm{H}, \mathrm{s}, \mathrm{H}_{9,19}\right), 6.96(2 \mathrm{H}, \mathrm{d}, J=8 \mathrm{~Hz}$, $\left.\mathrm{H}_{11,18}\right), 7.25\left(2 \mathrm{H}, \mathrm{dd}, J_{l}=J_{2}=8 \mathrm{~Hz}, \mathrm{H}_{12,19}\right) ; 7.52\left(2 \mathrm{H}, \mathrm{s}, \mathrm{H}_{7,14}\right), 9.60(2 \mathrm{H}, \mathrm{s}, 2 \mathrm{x}-\mathrm{OH}) ;{ }^{13} \mathrm{C}-\mathrm{NMR}(125 \mathrm{MHz}$, ppm, DMSO-d $\left.\mathrm{d}_{6}\right): \delta 22(\mathrm{~s}), 27(\mathrm{~s}), 116(\mathrm{t}), 121(\mathrm{t}), 129(\mathrm{t}), 135(\mathrm{t}), 136(\mathrm{t}), 136(\mathrm{t}), 157(\mathrm{q}), 189(\mathrm{C}=\mathrm{O}) ; \mathrm{MS}$ (EI-MS, m/z, \%): 308 ([M+2H],35), 306(100), 289(100), 277(12), 157(23), 145(20), 131(55), 115(64), 103(40), 91(32), 77(75), 51(20). Obtained as $\mathrm{C}_{20} \mathrm{H}_{18} \mathrm{O}_{3}$

\section{Antibacterial Assay}

The assay was performed by using the microdilution method, against Gram-negative and Gram-positive bacteria. This process is carried out in four steps, sterilization, preparation of bacteria inoculum, preparation of the compound solution and microdilution test. Sterilization is done with an autoclave at $121{ }^{\circ} \mathrm{C}$ for 20 minutes. Five Bacteria were prepared by using standard method ${ }^{29-30}$ and those bacteria are Staphylococcus aureus (S. aureus) ATCC 25923, Escherichia coli (E. coli) ATCC 25922, Bacillus subtilis (B. subtilis) ATCC 6633, Klebsiella pneumoniae (K. pneuminiae), and Enterococcus faecalis (E. faecalis) ATCC 29212. Solution of tested compounds were prepared in DMSO in concentration series as $100 \mu \mathrm{g} / \mathrm{mL} ; 50$ $\mu \mathrm{g} / \mathrm{mL} ; 25 \mu \mathrm{g} / \mathrm{mL} ; 12.5 \mu \mathrm{g} / \mathrm{mL}$ and $6.25 \mu \mathrm{g} / \mathrm{mL}$. It was also prepared DMSO without the tested compound in it as solvent control, and amoxicillin at $25 \mu \mathrm{g} / \mathrm{mL}$ of concentration as a positive control. The microdilution tested was carried out according to CLSI. ${ }^{30}$

\section{RESULTS AND DISCUSSION}

The dibenzylydene-cyclohexanones were obtained in moderate to high yields. The synthesis was carried out according to carbonyl condensation between aromatic aldehyde and cyclohexanone like Scheme-1 below. The reaction used $\mathrm{HCl}$ as a catalyst and THF as a solvent. The reaction was easy to perform and able to scale up.<smiles>[R]c1c([R])c([R5])c(C=O)c([R5])c1[R5]</smiles><smiles>[R]c1c([R])c([R])c(/C=C2\CCC/C(=C\c3c([R3])c([R])c([R])c([R])c3[R])C2=O)c([R3])c1[R]</smiles>

\section{Dibenzylidenecyclohexanone}

Scheme-1: Synthesis of Dibenzylidenecy clohexanone

A111 has a high yield of around $81 \%$ where this compound has two methoxy (-OMe) groups in para position each other. While A108 gave yield only around 27\% where this compound has a formyl (-COH) group at meta position. The best yield was found when two methoxy (-OMe) groups were on the aromatic ring. Those compounds with ethoxy (-OEt), methoxy (-OMe), or hydroxy $(-\mathrm{OH})$ on their aromatic rings have better yield compared to those that have nitro or formyl group on their aromatic ring.

A102 was obtained in 58\% yield and did not show any significant activity as an antibacterial agent against K. pneumonia, E. coli ATCC 25292, S. aureus ATCC 25923, B. subtilis dan E. faecalis ATCC 29212 at $100-6.25 \mu \mathrm{g} / \mathrm{mL}$ of concentration. A103 was successfully synthesized in a $72.45 \%$ yield. The value of its MIC and MBC of the compound can not be determined (N/A), but it showed maximum growth of inhibition 
RASĀYAN J. Chem.

Vol. 14 | No. 3 |2090-2096| July - September | 2021

towards B. subtilis around 42,06\% at concentration $100 \mu \mathrm{g} / \mathrm{mL}$. A104 was obtained in 53\% yield. The antibacterial test at a concentration of $6,25-100 \mu \mathrm{g} / \mathrm{mL}$ showed that $\mathbf{A 1 0 4}$ inhibited the growth of E. coli, K. pneumoniae, B. subtilis, S. aureus, and E. faecalis, not significantly. A108 was synthesized in $27 \%$ and did not show any significant activity against $E$. coli, $S$. aureus, $B$. subtilis, $K$. pneumoniae, and B. subtilis at concentrations $100-6.25 \mu \mathrm{g} / \mathrm{mL}$. The highest inhibition number was observed at concentration $25 \mu \mathrm{g} / \mathrm{mL}$ against $B$. subtilis around $30 \%$. It means that the MIC and MBC cannot be determined. A111 was successfully synthesized with a yield of $81.02 \%$. The results of its antibacterial activity test on E. coli, $K$. pneumoniae, $S$. aureus, B. subtilis, and E. faecalis did not show any good antibacterial activities. A129 compound was synthesized in $80 \%$ yield. The result of its antibacterial activity test showed that the concentration of up to $100 \mu \mathrm{g} / \mathrm{mL}$ gave the activity of maximum inhibition growth around $20 \%$ against $K$. pneumonia, E. coli, $30 \%$ against $S$. aureus, E. faecalis, and $50 \%$ against $B$. subtilis bacteria. The moderate yield was obtained from the synthesis of A146. Its antibacterial assay showed that compound $\mathbf{A 1 4 6}$ has MIC and MBC values at $50 \mu \mathrm{g} / \mathrm{mL}$ in E. coli, S. aureus, and E. faecalis. Furthermore, it showed the antibacterial activity by observing the percentage of inhibition in E. coli and $S$. aureus $100 \%$, E. faecalis $88 \%$, B. subtilis $50 \%$, and K. pneumoniae $9.45 \%$ at $50 \mu \mathrm{g} / \mathrm{mL}$.

Among all the tested compounds, none is active as an antibacterial against $K$. pnemoniae bacteria in terms of giving inhibition around $50 \%$ and more. A146 successfully inhibited the growth of bacteria against $E$. coli at $25 \mu \mathrm{g} / \mathrm{mL}, S$. aureus at $50 \mu \mathrm{g} / \mathrm{mL}$, E. faecalis at $12.5 \mu \mathrm{g} / \mathrm{mL}$ and B. subtilis at $25 \mu \mathrm{g} / \mathrm{mL}$. It seems that the microdillution method as the chosen assay for the antibacterial activity is not suitable for these kinds of curcumin analogs. According to the structure-activity relationship, it is found that compound $\mathbf{A 1 4 6}$ has hydroxy $(-\mathrm{OH})$ group on the aromatic ring, has a better antibacterial activity compared to others.

Then this activity was explained by using its docking study which was carried out against 5 OYO protein target. The results were shown below (Fig.-1).

Among the tested compounds, compound $\mathbf{A 1 0 2}$ has the smallest score docking value $=-13.6521$. While compound A146 only has a score docking value $=-13.0232$ lower than the score docking value of A102 but both A102 and A146 have better score docking values compared to the score docking value of amoxicillin, score docking value $=-12.9697$. According to the docking study result, compound $\mathbf{A 1 0 2}$ is more potent compare to A146. From the experimental result, compound A146 is the most potent antibacterial agent among the tested compounds but it is still not good enough compared to amoxicillin.

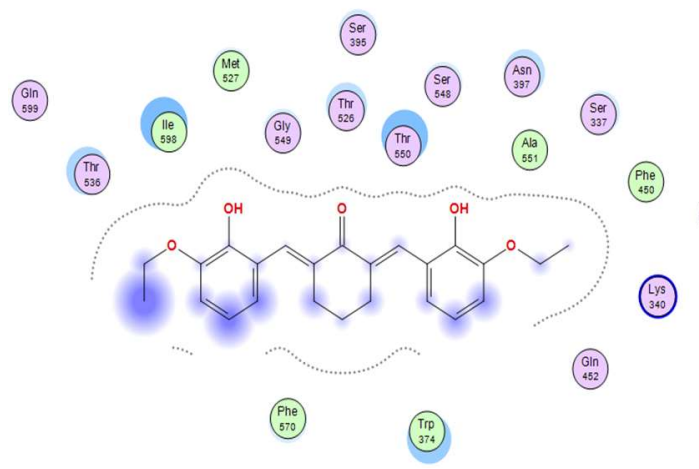

A102, $\mathrm{S}$ value $=-13.6521$

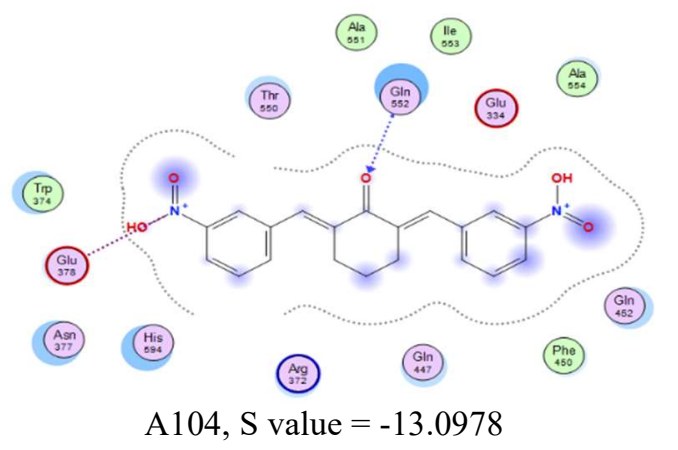

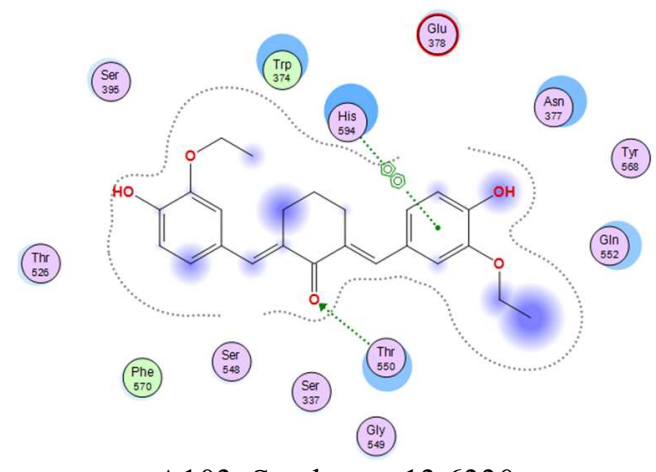

$\mathrm{A} 103, \mathrm{~S}$ value $=-13.6330$

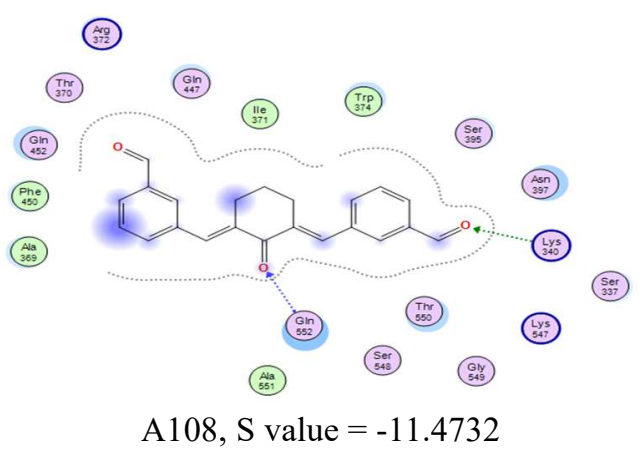

DIBENZYLIDENE-CYCLOHEXANONE 
RASĀYAN J. Chem.

Vol. 14 | No. 3 |2090-2096| July - September | 2021

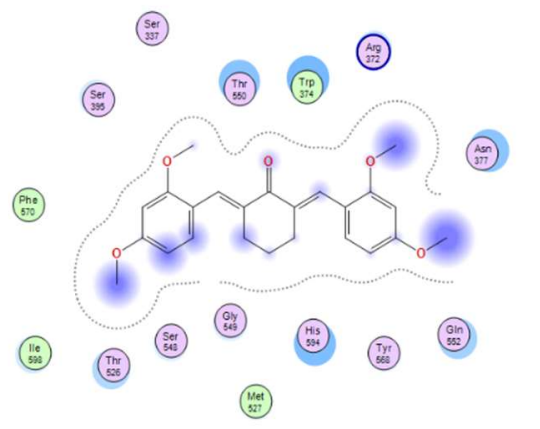

A111, S value $=-11.7930$

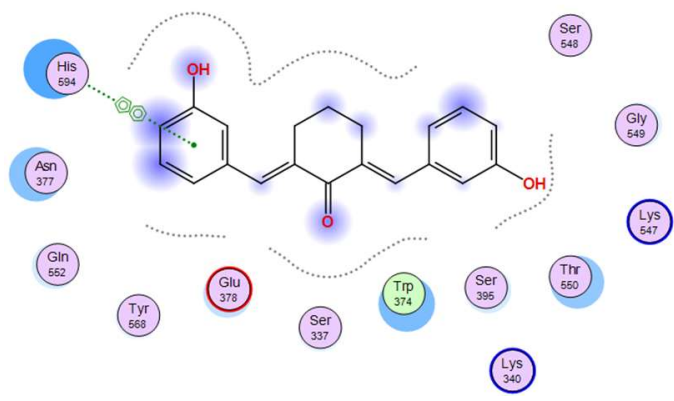

A146, S value $=-13.0232$

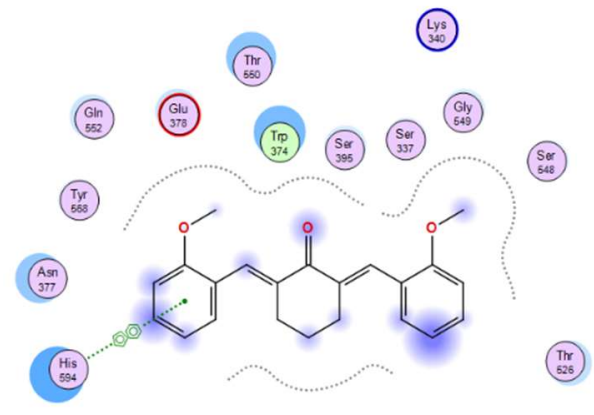

A129, $\mathrm{S}$ value $=-10.6779$

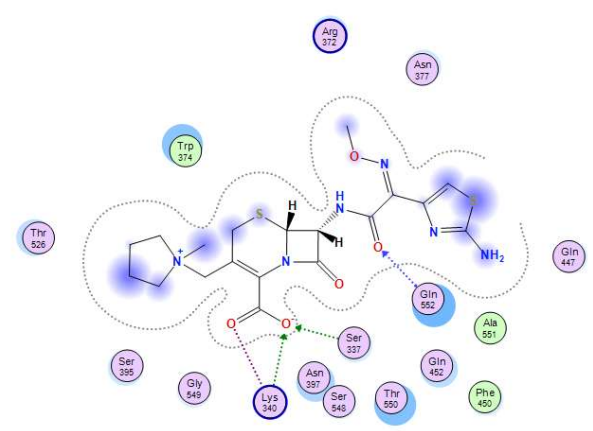

Amoxicillin, S value $=-12.9697$

Fig.-1: Docking Study of the tested Compounds

\section{CONCLUSION}

Compound A111, which has two methoxy groups on its aromatic ring, has the highest yield and compound A146, which has hydroxy group on its aromatic ring, is the most potent one according to the experiment but compound A102, which has ethoxy and hydroxy groups on its aromatic ring, has the smallest score docking which means that compound $\mathbf{A 1 0 2}$ should be the most potent one according to the molecular docking study but this was not approved by experiment.

\section{ACKNOWLEDGEMENT}

Thanks to Tesia Aisyah Rahmania for the technical work of docking study.

\section{REFERENCES}

1. S. Naz, S. Jabeen, S. Ilyas, F. Manzoor and F. Aslam, A. Ali, Pakistan Journal of Botany, 42, 455 (2010)

2. S-Y, Teow, K. Liew, S.A. Ali, AS-B. Khoo and S-C. Peh, Jornal of Tropical Medicine, ID 2853045, 10 pages (2016), https://doi.org/10.1155/2016/2853045

3. S.K. Kulkarni and A. Dhir, Indian Journal of Pharmaceutical Sciences, 72, 149(2010), https://doi.org/10.4103/0250-474X.65012

4. H.A.S. Murad, M.I. Suliaman, H. Abdallah and M. Abdulsattar, Indian Journal of Pharmaceutical Sciences, 76, 203(2014), https://doi.org/10.4103/0250-474X.134985

5. H.Y. Yow, N. Ahmad, N. Azmi, and M. Makmor-Bakry, Indian Journal of Pharmaceutical Sciences, 79, 267(2017), https://doi.org/10.4172/pharmaceutical-sciences.1000225

6. B. Antony, B. Merina, V.S. Iyer, N. Judy, K. Lennertz and S. Joyal, Indian Journal of Pharmaceutical Sciences, 70, 445(2008), https://doi.org/10.4103/0250-474X.44591

7. P.K. Gogu and A.V. Jithan, Indian Journal of Pharmaceutical Sciences, 72, 346(2010), https://doi.org/10.4103/0250-474X.70481

8. E.V. Rao and P. Sudheer, Indian Journal of Pharmaceutical Sciences, 73, 262(2011), https://doi.org/10.4103/0250-474X.93508

9. E.V. Rao, Y.R. Prasad, P. Sudheer, Indian Journal of Pharmaceutical Sciences, 79, 820(2017), https://doi.org/10.4172/pharmaceutical-sciences.1000296 
10. K. Girish, B.C. Channu and A.R. Baba, Indian Journal of Pharmaceutical Sciences, 81, 150(2019), https://doi.org/10.4172/pharmaceutical-sciences.1000491

11. S.C. Gupta, S. Prasad, J.H. Kim, S. Patchva, L.J. Webb, I.K. Priyadarsini and B.B. Aggarwal, Natural Product Report, 28, 1937(2011), https://doi.org/10.1039/c1np00051a

12. R.K. Maheshwari, A.K. Singh, J. Gaddipati and R.C. Srimal, Life Science, 78, 2081(2006), https://doi.org/10.1016/j.lfs.2005.12.007

13. S.Z. Moghadamtousi, H.A. Kadir, P. Hassandarvish, H. Tajik, S. Abubakar and K. Zandi, Biomedical Research International., ID186864 (2014), https://doi.org/https://doi.org/10.1155/2014/186864

14. C.I.N.H. Safitri, Ritmaleni, N. Rintiswati, Sardjiman and T. Kaneko, Asian Journal of Pharmaceutical and Clinical Research, 11(4), 226(2018), https://doi.org/10.22159/ajpcr.2018.v11i4.22991

15. C.I.N.H. Safitri, Ritmaleni, N. Rintiswati, Sardjiman and T. Kaneko, IOSR Journal of Dental and Medical Sciences, 16(2), 21(2017), https://doi.org/10.9790/0853-1612062126

16. O. Ramayanti, M. Brinkkemper, S. Verkuijlen, L. Ritmaleni, M.L. Go and J. Middeldorp, Cancers, 10(4), 89(2018), https://doi.org/10.3390/cancers10040089

17. M.V. Aanandhi, K. Gnanaprakash, M. Chandrakar, R.K. Raj, P. Shanmugasundaram, Rasayan Journal of Chemistry, 2(2), 375(2009)

18. S.K. Jain, M.S. Gill, H.S. Pawar and S. Suresh, Indian Journal of Pharmaceutical Sciences,76, 415(2014), https://doi.org/10.4103/0250-474X.143096

19. P. Jayasekhar, S.B. Rao and G. Santhakumari, Indian Journal of Pharmaceutical Sciences, 64, 82(2002)

20. O.A. Hamed, N. Mehdawi, A.A. Taha, E.M. Hamed, M.A. Al-Nuri and A.S. Hussein, Iranian Journal of Pharmaceutical Sciences, 12, 47(2013)

21. G. Liang, S. Yang, L. Jiang, Y. Zhao, L. Shao, J. Xiao and F. Ye, Chemical and Pharmaceutical Bulletin, 56, 162(2008), https://doi.org/10.1248/cpb.56.162

22. B. Wijianto, R. Ritmaleni, H. Purnomo, A. Nurrochmad, Rasayan Journal of Chemistry, 13(2), 1153(2020), http://dx.doi.org/10.31788/RJC.2020.1325554

23. T.A. Rahmania, R. Ritmaleni and E.P. Setyowati, Journal of Applied Pharmaceutical Science, 10(3), 039(2020), https://doi.org/10.7324/JAPS.2020.103004

24. S. Chhibber, S. Bansal and S. Kaur, Microbiology, 161, 1369(2015), https://doi.org/10.1099/mic.0.000104

25. Ritmaleni, T.A. Rahmania, P.N. Damayanti, E.P. Setyowati, International Journal of Pharmaceutical Research, 13(1), 3961(2021), https://doi.org/10.31838/ijpr/2021.13.01.543

26. Y. He, W. Li, G. Hu, H. Sun, Q. Kong, Frontiers in Oncology, 8, Article 614(2018), https://doi.org/10.3389/fonc.2018.00614

27. W. Li, Y. He, R. Zhang, G. Z, D. Zhou, Aging, 11(2), 771(2019), https://doi.org/10.18632/aging.101787

28. A.H. Cahyana, B. Ardiansah, W.H. Ibrahim, Rasayan Journal of Chemistry, 12(2), 471(2019), https://doi.org/http://dx.doi.org/10.31788/RJC.2019.1225011

29. S. Kolarevic, D. Milovanovic, M. Avdovic, M. Oalde, J. Kostic, K. Sunjog, B. Nikolic, J. KnezevicVukcevic, B. Vukovic-Gacic, Botania Serbica, 40, 29(2016), https://doi.org/10.5281/zenodo.48854

30. CLSI, Methods for Dilution Antimicrobial Susceptibility Tests for Bacteria that Grow Aerobically: Approved Standard-Ninth Edition, Clinical and Laboratory Standards Institute, Wayne; p. 18-20, (2012)

[RJC-6240/2020] 